\title{
A role for Ddc1 in signaling meiotic double-strand breaks at the pachytene checkpoint
}

\author{
Eun-Jin Erica Hong ${ }^{1}$ and G. Shirleen Roeder ${ }^{1-4}$ \\ ${ }^{1}$ Department of Molecular, Cellular and Developmental Biology, ${ }^{2}$ Department of Genetics, and ${ }^{3}$ Howard Hughes Medical \\ Institute, Yale University, New Haven, Connecticut 06520-8103, USA
}

\begin{abstract}
The pachytene checkpoint prevents meiotic cell cycle progression in response to unrepaired recombination intermediates. We show that Ddc1 is required for the pachytene checkpoint in Saccharomyces cerevisiae. During meiotic prophase, Ddc1 localizes to chromosomes and becomes phosphorylated; these events depend on the formation and processing of double-strand breaks (DSBs). Ddc1 colocalizes with Rad51, a DSB-repair protein, indicating that Ddc1 associates with sites of DSB repair. The Rad24 checkpoint protein interacts with Ddc1 and with recombination proteins (Sae1, Sae2, Rad57, and Msh5) in the two-hybrid protein system, suggesting that Rad24 also functions at DSB sites. Ddc1 phosphorylation and localization depend on Rad24 and Mec3, consistent with the hypothesis that Rad24 loads the Ddc1/Mec3/Rad17 complex onto chromosomes. Phosphorylation of Ddc1 depends on the meiosis-specific kinase Mek1. In turn, Ddc1 promotes the stable association of Mek1 with chromosomes and is required for Mek1-dependent phosphorylation of the meiotic chromosomal protein Red1. Ddc1 therefore appears to operate in a positive feedback loop that promotes Mek1 function.
\end{abstract}

[Key Words: Meiosis; pachytene checkpoint; cell cycle; recombination; Saccharomyces cerevisiae; phosphorylation]

Received August 15, 2001; revised version accepted November 30, 2001.

Checkpoints ensure the proper order of events in the cell cycle by inhibiting the initiation of late events until necessary early events are completed. Checkpoints also monitor the integrity of cellular structures. In the event of DNA damage, mitotic cell cycle progression is arrested or delayed by the DNA-damage checkpoint, thus providing time for repair. Mutations in checkpoints often have deleterious consequences such as cell lethality, genome instability, and cancer (for review, see Weinert 1998).

One of the first characterized checkpoint pathways in Saccharomyces cerevisiae is the DNA-damage checkpoint. Several proteins required for checkpoint function have been identified; these include $\operatorname{Rad} 24$ and the Ddc1 complex, which consists of Ddc1, Rad17, and Mec3 (for review, see Elledge 1996). Rad24 shares sequence homology with replication factor C (RFC; for review, see Zhou and Elledge 2000), a protein that recognizes DNA template/RNA primer hybrids during DNA replication (for review, see Tsurimoto 1998). The Ddc1 complex has structural homology to proliferating-cell nuclear antigen

${ }^{4}$ Corresponding author.

E-MAIL shirleen.roeder@yale.edu; FAX (203) 432-3263.

Article and publication are at http://www.genesdev.org/cgi/doi/10.1101/ gad.938102.
(PCNA) (Venclovas and Thelen 2000), which clamps onto DNA and confers processivity to DNA polymerases $\delta$ and $\varepsilon$ (for review, see Tsurimoto 1998). The homology of Rad24 and the Ddc1 complex to DNA-binding proteins and the interaction between $\operatorname{Rad} 24$ and RFC subunits (Naiki et al. 2000) led to the hypothesis that Rad24 and the Ddcl complex are the sensors of DNA damage (for review, see Zhou and Elledge 2000). Rad24 is postulated to recognize DNA lesions and then recruit the Ddc1 complex to generate checkpoint signals (for review, see Zhou and Elledge 2000).

A pathway similar to the DNA-damage checkpoint operates in meiosis (for review, see Roeder and Bailis 2000), a special type of cell division in which diploid cells produce haploid progeny. This so-called pachytene checkpoint monitors DNA lesions that are intrinsic to meiosis. Meiotic recombination initiates with double-strand breaks (DSBs) that are repaired using nonsister chromatids as templates (for review, see Roeder 1997). Unrepaired DSBs or subsequent recombination intermediates are presumed to activate the pachytene checkpoint.

The pachytene checkpoint has been well characterized in the zip1, dmc1, and hop2 mutants of budding yeast (for review, see Roeder and Bailis 2000). Zipl is a synaptonemal complex protein that holds homologous chromosomes close together along their lengths during the 
pachytene stage of meiotic prophase (Sym et al. 1993). In the zip1 mutant, the resolution of Holliday junctions is impaired, and some DSBs remain unrepaired (Storlazzi et al. 1996). Dmcl is a meiosis-specific homolog of the Escherichia coli RecA protein. In $d m c 1$ strains, repair of DSBs fails; as a consequence, DSBs become hyperresected to expose unusually long single-stranded (ss) tails (Bishop et al. 1992). In hop2 mutants, DSBs remain unrepaired and many chromosomes undergo synapsis with nonhomologous partners (Leu et al. 1998). Because of the accumulation of recombination intermediates, zip1, $d m c 1$, and hop2 strains fail to complete meiosis and arrest at the pachytene stage.

The pachytene checkpoint is not unique to budding yeast (for review, see Roeder and Bailis 2000). In mice, recombination defects conferred by mutations in DMC1, MSH5, and MLH1 result in meiotic arrest and removal of the arrested germ cells by apoptosis. In Caenorhabditis elegans, mutations in RAD51 or DMC1 prevent pachytene exit in oocytes. Similarly, a mutation in $s p n-B$, a homolog of DMC1, induces meiotic arrest in Drosophila oocytes. Thus, the pachytene checkpoint observed in yeast seems to be conserved in higher eukaryotes.

Mutations in a number of genes abolish the pachytene checkpoint in budding yeast. These include genes encoding the meiosis-specific proteins Mek1 and Red1. Mek1 is a kinase that phosphorylates Red1, a component of the cores of meiotic chromosomes (Smith and Roeder 1997; Bailis and Roeder 1998). Mek1-mediated phosphorylation is reversed by the Glc7 phosphatase (Bailis and Roeder 2000). Several observations suggest that Mek1dependent phosphorylation of Red 1 is necessary for arrest at the pachytene checkpoint (Bailis and Roeder 2000). Conversely, reversal of Mek1-mediated phosphorylation of Red1 (and/or other substrates) appears to be necessary for exit from the pachytene stage (Bailis and Roeder 2000).

The pachytene checkpoint also requires a subset of proteins that function in the mitotic DNA-damage checkpoint, namely, $\operatorname{Rad} 24, \operatorname{Rad} 17, \mathrm{Mec} 3, \mathrm{Ddc1}$, and the Mecl kinase (Lydall et al. 1996; this study; A. Hudson and G.S. Roeder, unpubl.). Mek1 fails to be phosphorylated in the absence of Mec1, $\operatorname{Rad} 24$, and Rad17, placing these proteins upstream of Mek1 (Bailis and Roeder 2000). Ddc1 and Mec3 have been tentatively placed downstream of Mek1 because Ddc1 and Mec3 are dispensable for Mek1 phosphorylation (Bailis and Roeder 2000).

In this work, we have examined the role of Ddc1 at the pachytene checkpoint. We show that Ddc1 localizes to meiotic chromosomes, and the number of Ddc1 foci correlates well with the presence of early intermediates in DSB repair. Ddc1 localization to meiotic chromosomes depends on the formation and processing of DSBs to generate ssDNA. Colocalization of Ddc1 with Rad51 suggests that Ddc1 is at the sites of DSB repair. In addition, Rad24 interacts with Ddc1 and with various recombination proteins, indicating that $\operatorname{Rad} 24$ also functions at DSB sites. Ddc1 localization depends on Rad24 and Mec3, supporting the idea that Rad24 loads the Ddc1 complex onto chromosomes. We have also determined the order of action of Ddcl with respect to Mek1 and Red1. Mek1 is required for phosphorylation of Ddc1, but not for its chromosomal localization. In turn, Ddc1 promotes the stable association of Mek1 with chromosomes and is required for Mek1-dependent phosphorylation of Red1. Thus, Ddc1 appears to act in a positive feedback loop that ensures the proper action of Mek1. In summary, we propose that $\operatorname{Rad} 24$ and the Ddc1 complex function at the sites of DSB repair, and we precisely position Ddc1 with respect to Mek1.

\section{Results}

Ddc1 is required for the pachytene checkpoint

A screen for mutations that allow the zip1 mutant to undergo meiotic nuclear division identified a mutation in the DDC1 gene (see Materials and Methods). The ddc1 mutation completely abolishes arrest in zip1; the zip1 ddc1 double mutant undergoes nuclear division with wild-type kinetics and frequency (Fig. 1A). The $d d c 1$ mutation also alleviates meiotic arrest in $d m c 1$ and hop2. However, the frequency of nuclear division in the $d m c 1 d d c 1$ and hop2 ddc1 double mutants does not reach the wild-type level, and sporulation is delayed compared with wild type (Fig. 1B,C).

In principle, the ddc1 mutation could allow zip1/ dmc1/hop2 to undergo nuclear division either by suppressing the defects in meiotic recombination or by inactivating the checkpoint that causes cells to arrest. To distinguish these possibilities, the presence of recombination intermediates was assayed indirectly by monitoring Rad51 foci. $\operatorname{Rad51}$ (a RecA homolog) is a recombination protein that localizes to meiotic chromosomes (Bishop 1994). Rad51 foci have been used as a marker for ongoing DSB repair (Lydall et al. 1996). During meiosis in wild type, Rad51 foci dissociate from chromosomes when recombination is completed, prior to the first meiotic division. However, staining of surface-spread nuclei from hop2 ddc1 cells with antibodies to Rad51 and tubulin shows that abundant Rad51 foci remain on chromosomes even at the time of the meiosis I division (Fig. 1D). Thus, the $d d c 1$ mutation allows hop2 to undergo chromosome segregation despite the presence of recombination intermediates, indicating that $d d c 1$ disrupts the pachytene checkpoint.

Two additional observations indicate that $d d c 1$ disrupts the pachytene checkpoint in zip1 rather than suppressing the meiotic defects. First, the zip1 ddc1 double mutant does not make a synaptonemal complex. Staining chromosome cores with antibodies to Red1 shows that homologs are not intimately synapsed /data not shown), as is the case for the zip1 single mutant. Second, $d d c 1$ does not improve spore viability in zip1. The zip1 mutant shows a spore viability of $44 \%$ (cf. $94 \%$ in wild type) because of reduced crossing over and consequent homolog nondisjunction at meiosis I. The ddc1 cells have $53 \%$ spore viability, whereas zip1 $d d c 1$ cells have $12 \%$ spore viability. The zip1-dependent reduction in 

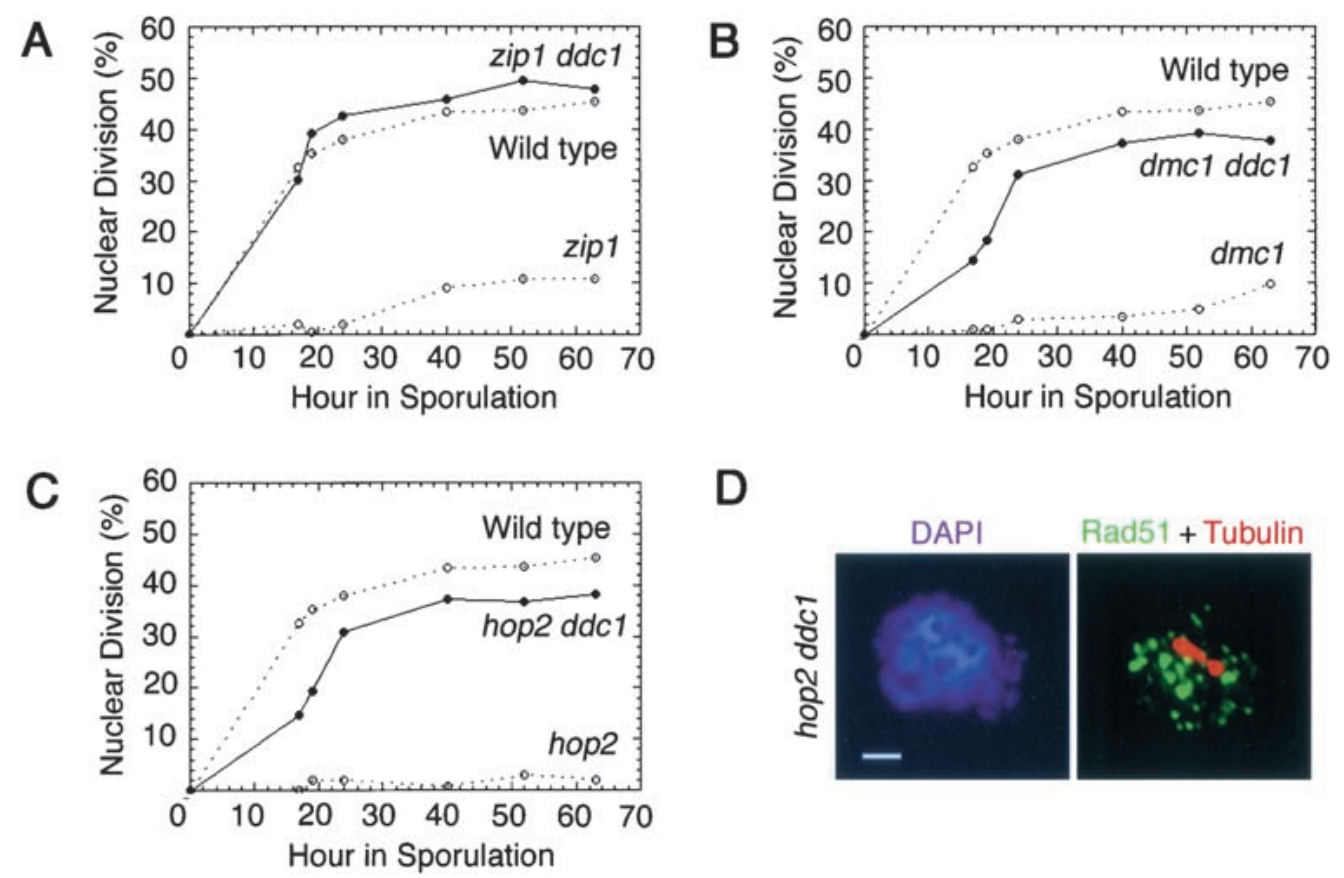

Figure 1. Ddc1 is required for the pachytene checkpoint. A mutation in DDC1 allows $(A)$ zip1, $(B)$ dmc1, and $(C)$ hop2 to undergo meiotic nuclear divisions. Both binucleate and tetranucleate cells were scored as having undergone nuclear division. These time courses were repeated at least three times with qualitatively similar results. (D) Meiotic chromosomes from the hop2 ddc1 strain were surface-spread and stained with a DNA-binding dye (DAPI; blue) and with antibodies to Rad51 (green) and tubulin (red). Cells undergoing meiosis I were identified by the presence of an elongated spindle. Bar, $2 \mu \mathrm{m}$.

spore viability in $d d c 1$ cells implies that the zip1 defects in recombination and chromosome segregation are still manifest in the absence of Ddc1.

\section{Ddc1 is hyperphosphorylated in checkpoint-arrested mutants}

Ddc1 is phosphorylated in response to DNA damage in the mitotic DNA-damage checkpoint (Longhese et al. 1997). To test whether Ddc1 is regulated by phosphorylation in meiosis, a gel shift assay was carried out. A modified version of the $D D C 1$ gene encoding a protein tagged with three copies of the myc epitope was used to replace the chromosomal $D D C 1$ gene. The $D D C 1-m y c$ gene is fully functional; it does not confer sensitivity to ultraviolet irradiation, nor does it disrupt meiotic arrest in zip1 strains (data not shown). Furthermore, DDC1myc strains show $96 \%$ spore viability, compared with $94 \%$ in wild-type and $53 \%$ in ddc1 strains. Ddc1-myc protein was immunoprecipitated from meiotic cell extracts and analyzed by Western blotting.

During meiotic prophase in wild-type cells, the Ddc1 protein is heterogeneous in electrophoretic mobility, with a significant fraction of the protein migrating slower than the predominant band (Fig. 2A). The slowermigrating forms are expected if the protein is phosphorylated. To test whether phosphorylation of Ddc1 correlates with checkpoint activation, checkpoint-arrested mutants were analyzed. Ddc1 becomes hyperphosphory- lated in zip1, dmc1, and hop2 strains (Fig. 2A), showing that the checkpoint regulates the extent of Ddc1 phosphorylation. In addition, the amount of phosphorylated Ddc1 protein correlates with the severity of the arrest; there is more hyperphosphorylated Ddc1 in hop2 than in zip1 (Fig. 2A) cells, and hop2 cells undergo tighter arrest than do zip1 cells (Fig. 1).

To confirm that the slower migration of Ddc1 in meiosis is caused by phosphorylation, the Ddcl precipitate from $d m c 1$ cells was treated with calf intestinal phosphatase (CIP). CIP treatment transformed the slower-migrating forms of Ddc1 to a single faster-migrating form (Fig. 2B). This change in mobility was prevented when a phosphatase inhibitor was added together with CIP, showing that the shift in Ddc1 mobility in meiosis is caused by phosphorylation.

Hyperphosphorylation of Ddc1 in zip1, dmc1, and hop2 cells could be an indirect consequence of arrest at a stage in the cell cycle when Ddc1 is normally phosphorylated, rather than a direct consequence of checkpoint activation. To test this possibility, Ddc1 phosphorylation was analyzed in the $n d t 80$ mutant. ndt 80 cells arrest at the pachytene stage, independent of the pachytene checkpoint, because genes required for the exit from pachytene are not expressed (Chu and Herskowitz 1998). Ddc1 phosphorylation in $n d t 80$ cells occurs at a low level, comparable to that seen in wild-type cells (Fig. 2A), indicating that the hyperphosphorylation observed in checkpoint-arrested mutants is not simply owing to cell cycle arrest at pachytene. 


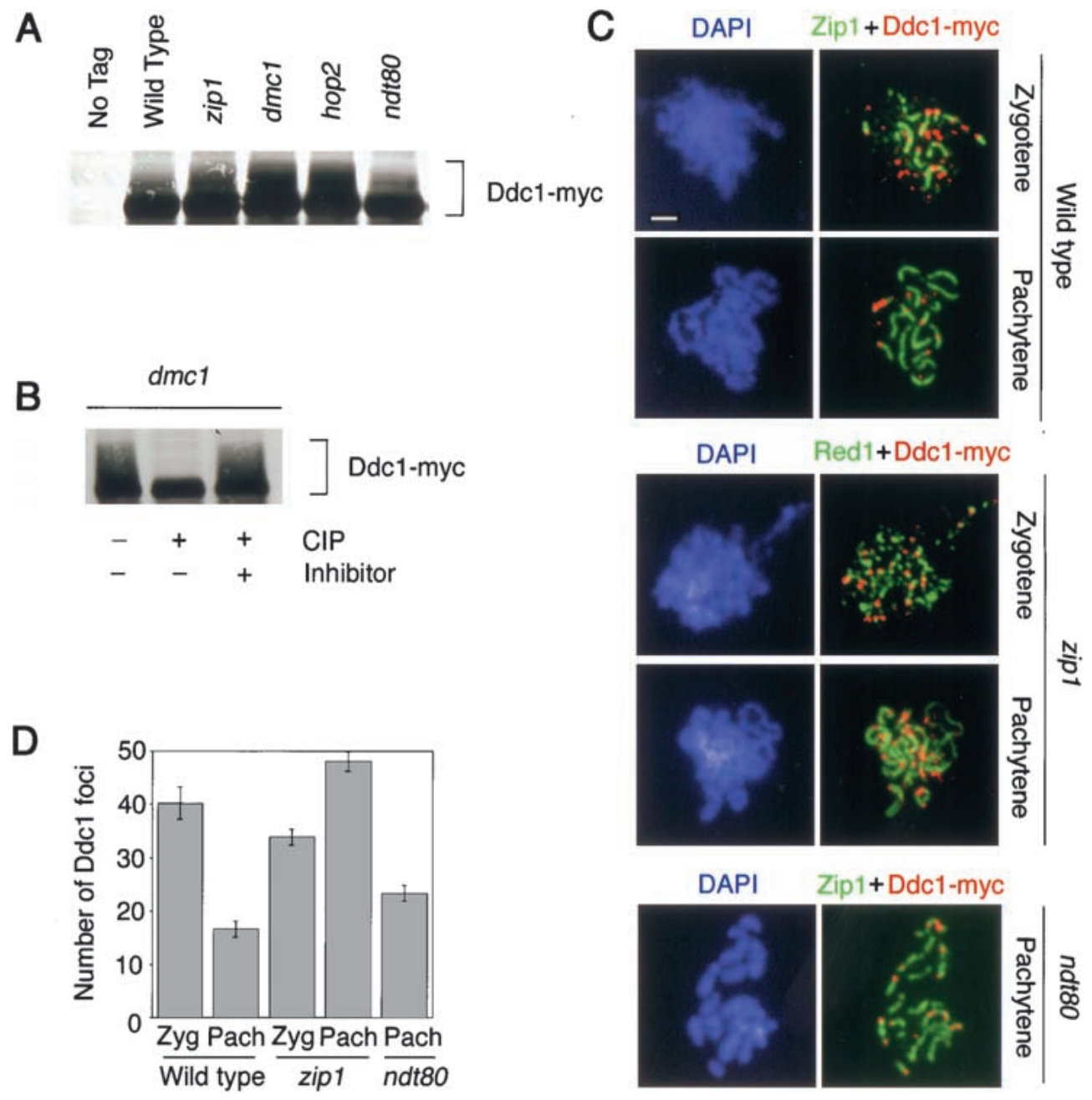

Figure 2. Ddc1 becomes phosphorylated and localizes to meiotic chromosomes. $(A)$ Immunoprecipitation and Western blot analysis of Ddc1-myc after $15 \mathrm{~h}$ of sporulation, when most cells are at the pachytene stage. $(B)$ CIP treatment of the Ddc1-myc precipitate transforms the slower-migrating forms to a single faster-migrating form. CIP inhibitor, $\beta$-glycerolphosphate, was used at $5 \mathrm{mM}$. (C) Spread meiotic nuclei stained with DAPI (blue), antibodies to Zip1 or Red1 (green), and antibodies to Ddc1-myc (red). Bar, $2 \mu \mathrm{m}$. (D) Quantitation of Ddc1 foci on meiotic chromosomes. An error bar represents the standard error. (Zyg) Zygotene; (Pach) pachytene.

\section{Ddc1 localizes to meiotic chromosomes and} accumulates in checkpoint-arrested mutants

To test whether Ddc1 associates with meiotic chromosomes, nuclei from strains homozygous for $D D C 1-m y c$ were surface-spread and stained with antibodies to the myc epitope and to Zip1. Zip1 staining was used to identify cells at different stages of meiotic prophase. Zip1 localizes to chromosomes as dots and short linear stretches in zygotene, when chromosome synapsis is initiating. At pachytene, when chromosomes are fully synapsed, Zip1 localizes continuously along the length of each chromosome pair (Sym et al. 1993).

In wild-type cells, Ddc1 localizes to meiotic chromosomes as distinct foci; there are $\sim 40$ dots per nucleus in zygotene (Fig. 2C,D), when early intermediates of recombination are abundant (for review, see Roeder 1997). At pachytene, when synapsis is complete and most DSBs are repaired to form joint molecules (for review, see Roeder 1997), there is a twofold decrease in the number of Ddc1 foci on chromosomes (Fig. 2C,D). In addition, many Ddc1 foci are at telomeres at the pachytene stage (Fig. 2C).

To investigate whether the abundance of Ddc1 foci on meiotic chromosomes correlates with checkpoint activation, Ddc1 foci were quantitated in the zip1 mutant (Fig. 2C,D). At zygotene, there is a similar number of Ddc1 foci in spread chromosomes of zip1 and wild-type cells. However, at pachytene, there is an approximately threefold increase in the number of Ddc1 foci in zip1 compared with wild-type cells. This increase in Ddc1 foci is not an artifact of being arrested at pachytene, because the increase in Ddc1 foci is not seen in the ndt80 mutant (Fig. 2C,D). 
Ddc1 localization and phosphorylation depend on formation and processing of DSBS

Recombination proteins like Rad51 and RPA require the formation and processing of DSBs for their localization to meiotic chromosomes (Gasior et al. 1998). To determine whether Ddcl phosphorylation and localization show a similar dependence, Ddcl foci were quantitated, and phosphorylation was assessed in mutants defective at different steps in the recombination process.

In the spo11 mutant, DSB formation is eliminated (for review, see Roeder 1997). Ddc1 foci are almost completely abolished in spo11 cells (Fig. 3B,C), and the phosphorylation of Ddc1 is eliminated (Fig. 3A).

In the rad50S strain, DSB formation is normal, but resection of DSBs to expose ssDNA is prevented (for review, see Roeder 1997). Ddc1 localization is significantly impaired in rad50S strains. This defect is most pronounced at the zygotene stage (Fig. 3B,C); the number of foci approaches the wild-type level by pachytene (data not shown). The level of Ddc1 phosphorylation in rad50S is similar to wild type (Fig. 3A); it is substantially less than the level of phosphorylation observed in $d \mathrm{mc1}$ strains, which accumulate a similar number of unre-

A

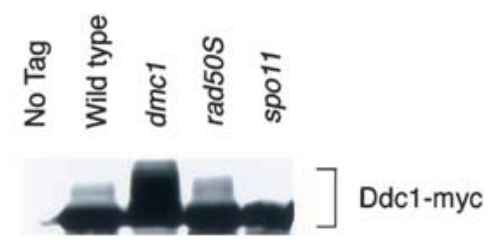

B

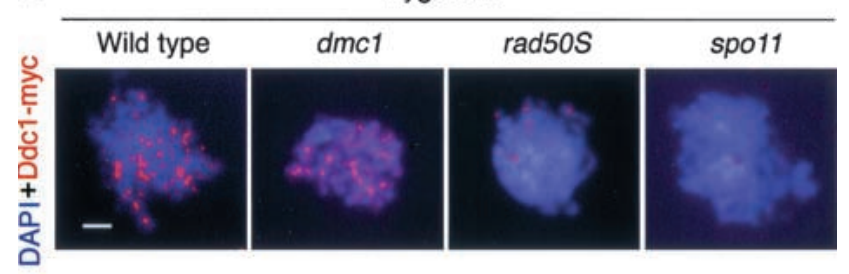

C

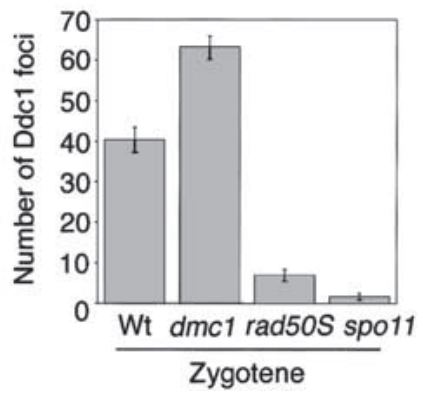

Figure 3. Ddcl phosphorylation and localization depend on formation and processing of DSBs. (A) Western blot analysis of Ddc1-myc in mutants defective in the formation and processing of DSBs at $15 \mathrm{~h}$ in meiosis. (B) Ddc1 localization to meiotic chromosomes in strains lacking DSBs (spo11) or DSB-associated ssDNA (rad50S) and in the dmc1 mutant, which accumulates DSBs. DAPI (blue); Ddc1-myc (red). Bar, $2 \mu \mathrm{m}$. (C) Quantitation of Ddc1 foci on meiotic chromosomes. An error bar represents the standard error. (Wt) Wild type. paired (albeit processed) DSBs. In the sae2 strain, another mutant in which DSBs remain unprocessed (for review, see Roeder 1997), the levels of Ddc1 localization and phosphorylation are similar to those observed in rad50S cells (data not shown). The rad50S and sae2 data suggest that processed DSBs are required for normal localization of Ddc1 to chromosomes.

\section{Localization of Ddc1 to meiotic chromosomes depends on Rad24 and Mec3}

Rad24 is postulated to load the Ddc1 complex onto chromosomes (for review, see Zhou and Elledge 2000). Consistent with this hypothesis, Ddc1 interacts with Rad24 in the two-hybrid system in both vegetative and meiotic cells (Fig. 4A). The meiotic interaction is increased threefold when the pachytene checkpoint is activated by a hop2 mutation (Fig. 4A).

To test whether Ddc1 localization to chromosomes depends on Rad24 and the integrity of the Ddc1 complex, Ddc1 foci were quantitated in zip1 rad24 and zip1 mec3 strains. The zip1 background was chosen so that the pachytene checkpoint would be activated, thus enhancing Ddc1 phosphorylation and localization. Ddc1 localization is eliminated in zip1 mec3 and greatly reduced in zip1 rad24 cells (Fig. 4B,C). Accordingly, Ddc1 phosphorylation is also abolished in the absence of Rad24 and Mec3 (Fig. 4D).

\section{Ddc1 colocalizes with a DSB-repair protein}

The requirement of DSBs for Ddc1 localization to chromosomes raises the possibility that Ddc1 is at the sites of DSB repair. To test this possibility, meiotic chromosomes from hop2 cells (in which DSBs remain unrepaired) were surface-spread and costained with antibodies to Ddc1-myc and Rad51. In the hop2 cells, there is significant colocalization between Ddc1 and Rad51 (Fig. $5 \mathrm{~A}):=77 \% \pm 3 \%$ of Ddc1 foci colocalize with Rad51, whereas $77 \% \pm 3 \%$ of Rad51 foci colocalize with Ddc1. In contrast, the expected frequency of fortuitous overlap is only $16 \%$. The relative intensity of Rad51 to Ddc1 staining varies among foci, perhaps suggesting that maximum localization of these proteins occurs at different times in the DSB repair process. In wild-type cells, Rad51 does localize to foci on chromosomes (Bishop 1994), but there is no significant colocalization of Rad51 and Ddc1 (data not shown).

\section{Rad24 interacts with recombination proteins}

Additional evidence that Rad24 and (by implication) the Ddc1 complex are present at sites of DSB repair was obtained by two-hybrid protein analysis. Rad24 was fused to the Gal4 transcriptional activation domain, and various known recombination proteins were fused to the Gal4 DNA-binding domain. Rad24 was found to interact with Sae1, Sae3, and Rad57 (Fig. 5B,C,E), three proteins required for meiotic DSB repair (McKee and Kleckner 

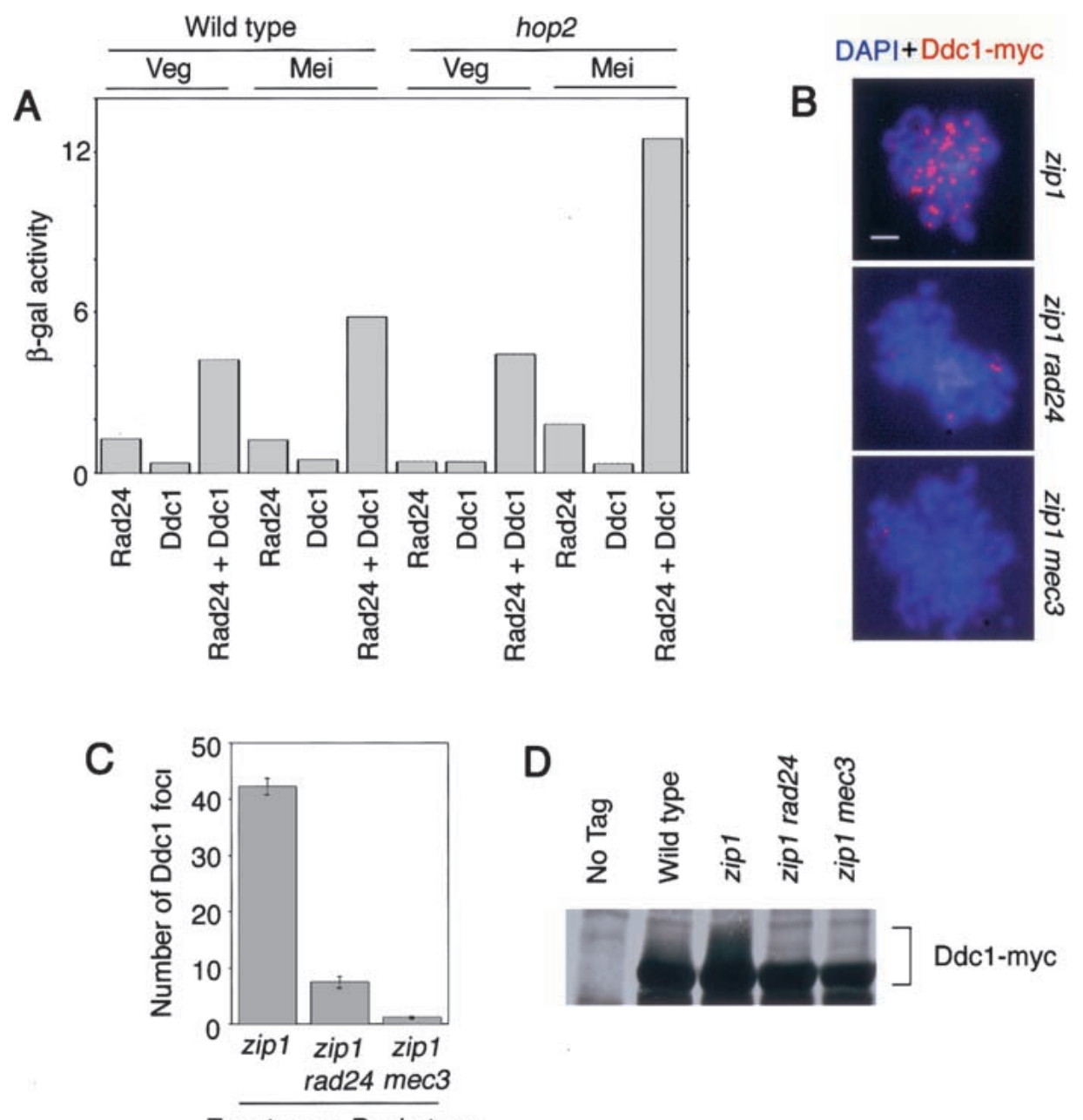

D

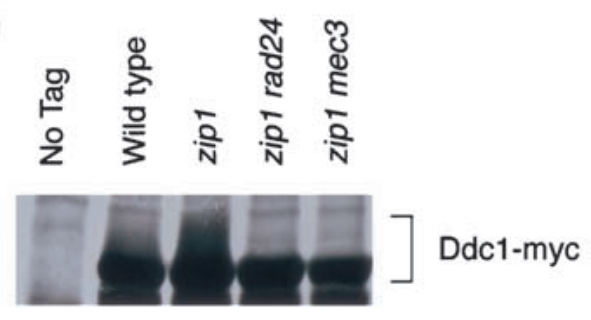

Zygotene + Pachytene

Figure 4. Ddc1 interacts with Rad24, and Ddc1 localization and phosphorylation depend on Rad24 and Mec3. (A) Two-hybrid interaction between Ddc1 and Rad24. Wild-type and hop2 cells in both vegetative and meiotic conditions were used for the assay. ( $\beta$-gal) $\beta$-Galactosidase; (Veg) vegetative; (Mei) meiotic. (B) Spread meiotic chromosomes stained with DAPI (blue) and antibody to Ddc1-myc (red). Bar, $2 \mu \mathrm{m}$. (C) Quantitation of Ddc1 foci on meiotic chromosomes from strains lacking Rad24 and Mec3. An error bar represents the standard error. (D) Immunoblot of Ddc1-myc in strains lacking Rad24 and Mec3 at $15 \mathrm{~h}$ in meiosis.

1997; for review, see Roeder 1997). In addition, Rad24 interacts with the meiosis-specific Msh5 protein (Fig. $5 \mathrm{D})$, which is required for wild-type levels of crossing over (for review, see Roeder 1997). Rad24 interacts with Sae1, Sae3, and Msh5 in both vegetative (Fig. 5B-D) and meiotic (data not shown) cells. However, the interaction between Rad24 and Rad57 is observed only in cells undergoing meiosis (Fig. 5E).

\section{Mek1 is required for Ddc1 phosphorylation} but not its localization

The dispensability of Ddc1 for Mek1 phosphorylation (Bailis and Roeder 2000) places Ddc1 either downstream of Mek1 or in a parallel pathway to Mek1. To position Ddc1 with respect to Mek1, Ddc1 phosphorylation in the zip1 mek1 double mutant was analyzed by a gel shift assay. Ddc1 phosphorylation is eliminated in zip1 mek1 and in zip1 mek1 ndt80 cells (Fig. 6A), placing Ddc1 downstream of Mek1.

To understand better the relationship between Ddc1 and Mek1, colocalization of Ddc1 and Mek1 on meiotic chromosomes was assayed. Mek1 was tagged with green fluorescent protein (GFP; see Materials and Methods), and the presence of Mek1 and Ddc1 was detected using antibodies to GFP and the myc epitope, respectively. Significant colocalization of Mek1 and Ddc1 is observed in hop2 cells (Fig. 6B), where Ddc1 undergoes hyperphosphorylation, but not in wild-type cells (data not shown), where Ddc1 phosphorylation is minimal. In hop2 cells, $82 \% \pm 2 \%$ of Ddc1 foci colocalize with Mek1, and $75 \% \pm 1 \%$ of Mek1 foci colocalize with Ddc1. The expected frequency of fortuitous colocalization of Mek1 and Ddc1 is only $12 \%$.

To determine whether Mek1 is required for proper localization of Ddc1, Ddc1 foci were quantitated in the 
A
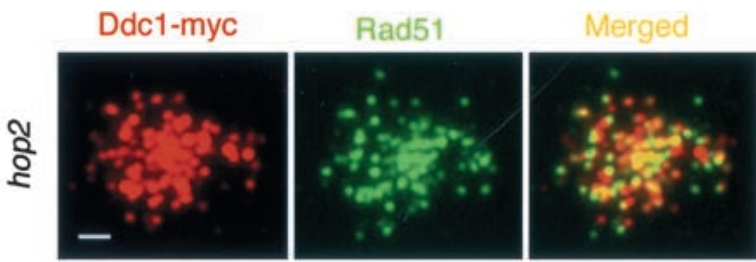

B

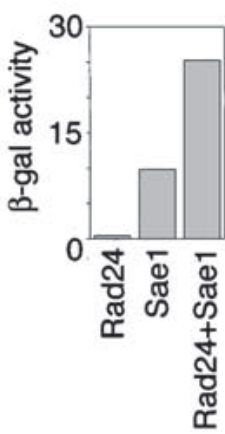

C

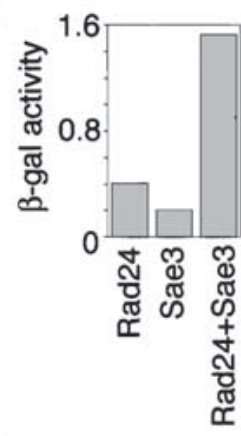

D

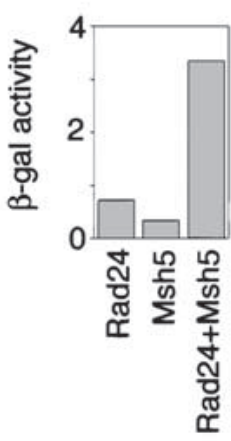

E

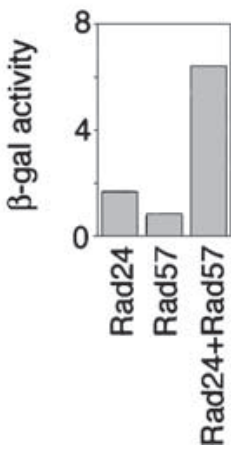

Figure 5. Ddc1 and Rad24 associate with sites of DSB repair. (A) Colocalization between Ddc1 (red) and Rad51 (green) on meiotic chromosomes from the hop2 strain. Overlapping foci appear yellow in the merged image; yellow is underrepresented owing to differences in the intensity of green and red foci. Bar, $2 \mu \mathrm{m}$. (B-E) Two-hybrid interaction between $\operatorname{Rad} 24$ and various recombination proteins: $(B)$ Sae1, $(C)$ Sae3, $(D)$ Msh5, and $(E)$ Rad57. Vegetative yeast cells were used for $B-D$, whereas meiotic cells were used for $E$. ( $\beta$-gal) $\beta$-Galactosidase.

zip1 mek1 double mutant. Although the number of Ddc1 foci is slightly reduced, Ddc1 localization to chromosomes is largely unaffected in zip1 mek1 cells (Fig. $6 \mathrm{C}, \mathrm{D})$.

\section{Red1 is required for Ddc1 localization to chromosomes}

To position Ddcl with respect to Red1 in the checkpoint pathway, the dependence of Ddc1 localization on Red1 was tested. Red1 phosphorylation is not required for Ddc1 localization to chromosomes, because Ddc1 localization is largely unaffected in zip1 mek1 cells (Fig. 6C), where Red1 phosphorylation is eliminated (Bailis and Roeder 1998). However, Ddc1 localization is eliminated in zip1 red1 cells (Fig. 6C,E), indicating that the presence of the Red1 protein is important for Ddc1 localization. Red1 is probably also required for Ddc1 phosphorylation, because Ddc1 phosphorylation depends on Mek1, and Mek1 fails to be phosphorylated or localized to chromosomes in red1 cells (Bailis and Roeder 1998).

\section{Ddc1 and Mec3 promote Mek1-dependent} phosphorylation of Red1

Although Ddc1 and Mec3 are not required for Mek1 phosphorylation (Bailis and Roeder 2000), it is possible that they affect the proper action of Mek1. To test this hypothesis, Mek1-dependent phosphorylation of Redl

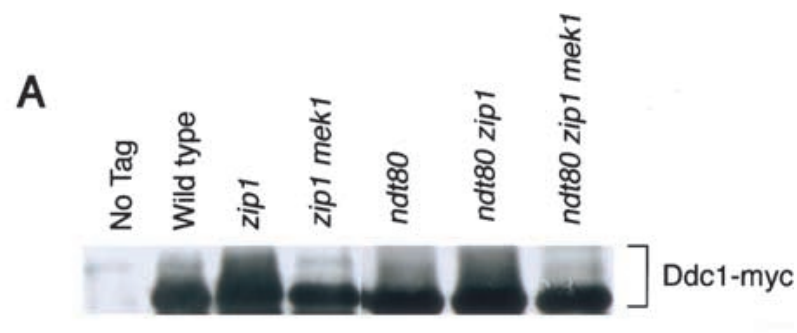

B
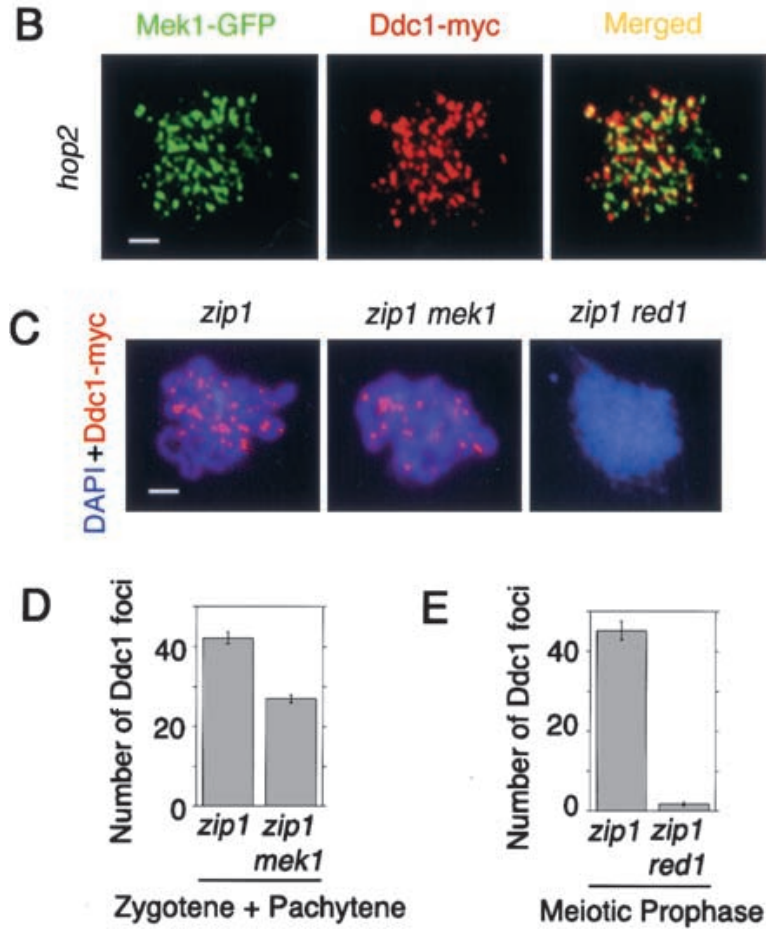

Figure 6. Phosphorylation, but not localization, of Ddc1 depends on Mek1. (A) Immunoblot of Ddc1-myc in strains lacking Mek1 at $15 \mathrm{~h}$ in meiosis. (B) Colocalization between Mek1-GFP (green) and Ddc1-myc (red). Overlapping foci appear yellow in the merged image; yellow is underrepresented owing to the differences in the intensity of green and red foci. $(C)$ Meiotic chromosomes from strains lacking Mek1 or Red1, stained with DAPI (blue) and antibody to Ddc1-myc (red). $(D, E)$ Quantitation of Ddc1 foci on meiotic chromosomes from strains lacking Mek1 $(D)$ or Red1 $(E)$. An error bar represents the standard error. Bars in $B$ and $C, 2 \mu \mathrm{m}$. 
was assessed by a gel shift assay. Red1 phosphorylation is eliminated in $d d c 1$ (data not shown) and $d m c 1 d d c 1$ ndt80 (Fig. 7A) cells. Similarly, Red1 phosphorylation is abolished in dmc1 mec3 ndt80 cells (Fig. 7A).

In principle, the failure of Red 1 phosphorylation in the ddc1 and mec3 mutants could be caused by inappropriate activation of Glc7, the phosphatase that normally dephosphorylates Red1. If so, then Red1 phosphorylation should be restored in strains lacking Glc7. To test this possibility, Red1 phosphorylation was assayed in $d m c 1$ ddc1 glc7 and dmc1 mec3 glc7 strains. The glc7 muta- tion had no effect on the level of Red1 phosphorylation (Fig. 7B).

Together, these results support the argument that Ddc1 and Mec3 affect the phosphorylation of Red1 by decreasing Mek1 kinase activity rather than by increasing Glc7 phosphatase activity.

Ddc1 is required for proper localization of Mek1

The failure of Red1 phosphorylation in ddc1 and mec3 mutants could be owing to a defect in Mek1 localization
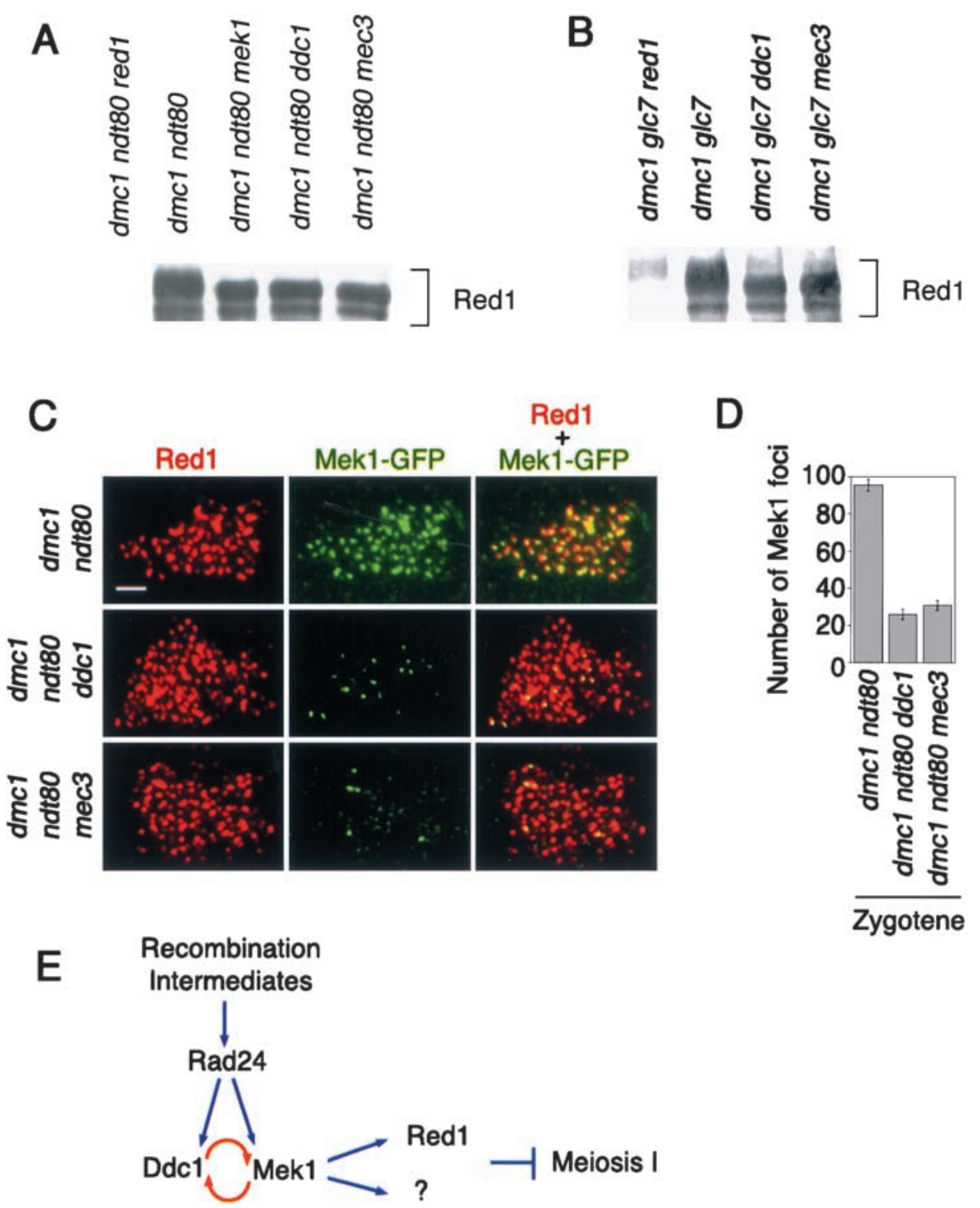

Figure 7. Mek1-dependent phosphorylation of Red1 requires Ddc1 and Mec3. (A) Immunoprecipitation and immunoblotting of Red1 in $d m c 1$ ndt80 strains lacking Ddc1 or Mec3. (B) Immunoprecipitation and immunoblotting of Red1 in $d m c 1$ glc7 strains lacking Ddc1 or Mec3. (C) Impaired Mek1 localization to meiotic chromosomes in dmc1 ndt80 strains lacking Ddc1 or Mec3. All strains are homozygous for the indicated mutations, except that $d m c 1$ ndt 80 is heterozygous for the $d d c 1$ disruption. Overlapping Red 1 (red) and Mek1 (green) foci appear yellow in the merged image. Bar, $2 \mu \mathrm{m}$. (D) Quantitation of Mek1 foci on meiotic chromosomes. An error bar represents the standard error. (E) Model of Ddc1 action; see text for details. 
to chromosomes. To explore this possibility, Mek1-GFP localization to meiotic chromosomes was assessed in dmc1 ndt80 strains lacking Ddc1 or Mec3. Mek1 localization is delayed and inefficient in $d m c 1 \mathrm{ndt} 80 \mathrm{ddc1}$ and $d m c 1$ ndt 80 mec3 strains; both the intensity and the number of Mek1 foci are decreased compared with those in $d m c 1 \mathrm{ndt} 80$ cells. The difference is most prominent in cells at zygotene (Fig. $7 \mathrm{C}, \mathrm{D}$ ). By pachytene, numerous Mek1 foci are on chromosomes, but the number and intensity do not reach the levels seen in $d m c 1$ ndt80 cells (data not shown). The Mek1 foci on chromosomes in $d d c 1$ strains both at zygotene and pachytene colocalize with Red1 (Fig. 7C), just as they do in wild type.

\section{Discussion}

\section{Ddc1 and Rad24 function at the sites of DSB repair}

Although many components of the DNA-damage and pachytene checkpoint pathways are known, their molecular functions remain largely unclear. Speculation has been based on the sequence and structural homology of the checkpoint molecules to previously characterized proteins. Rad24 shares sequence homology to RFC, and interacts with RFC subunits 2-5 (for review, see Zhou and Elledge 2000), proteins that recognize primed DNA during DNA replication. The Ddc1 complex shares structural homology to PCNA, a ring-shaped homotrimer that wraps around DNA and slides along the DNA duplex. PCNA, which requires RFC for its association with DNA, functions as a processivity factor for DNA polymerases by tethering them to DNA. It has been proposed that the Ddc1 complex forms a heterotrimeric PCNA-like structure that functions as a DNA-damagespecific PCNA aiding in repair synthesis of damaged DNA (Venclovas and Thelen 2000). These observations imply that Rad24 and the Ddc1 complex act at sites of DSB repair.

In an effort to place checkpoint proteins at the sites of DSBs, a number of mammalian checkpoint proteins have been localized to meiotic chromosomes (for review, see Roeder and Bailis 2000). These include the protein kinases Atr (a homolog of Mec1), Atm, and Chk1, as well as Rad1 (a homolog of S. cerevisiae Rad17). Neither Atr nor Rad1 was found to colocalize with Dmc1 or Rad51 (Atm and Chk1 were not tested).

In this work, we provide evidence that Ddc1 functions at the sites of DSB repair. Ddc1 shows substantial colocalization with Rad51 in mutant backgrounds in which DSBs remain unrepaired. Ddc1 localization to meiotic chromosomes requires the formation and processing of DSBs, and the number of Ddc1 foci on meiotic chromosomes correlates well with the abundance of early recombination intermediates in both wild-type and mutant strains. In addition, Rad24 interacts with Ddc1 and recombination proteins (Sae1, Sae3, Msh5, and Rad57) in the two-hybrid system. In meiotic cells, the Ddc1-Rad24 interaction is increased threefold when the pachytene checkpoint is activated. These findings suggest that
Ddc1 and Rad24 function together at the sites of DSB repair. Consistent with our two-hybrid data, the human homolog of Rad24 has been shown to coimmunoprecipitate with the human counterpart of the Ddc1 complex (Rauen et al. 2000).

Evidence for Ddc1 localization to DSBs in vegetative cells has been recently provided by Kondo et al. (2001), who used chromatin immunoprecitation to show that Ddc1 associates with DNA at the site of a DSB induced by the budding yeast $\mathrm{HO}$ endonuclease. Evidence that Rad24 also localizes to DSBs comes from the demonstration that the fission yeast Rad17 protein (a homolog of $S$. cerevisiae Rad24) associates with chromatin in response to ionizing irradiation (Kai et al. 2001). A point mutant of Rad17 (K118E) that is unable to bind ATP shows reduced binding to chromatin (Kai et al. 2001), consistent with the notion that ATP is required for RFC to open up the PCNA ring in order to load itself and the PCNA complex onto DNA (for review, see Tsurimoto 1998). If ATP binding is required solely for Rad17 to open up the Rad9/Rad1/Hus1 complex / the fission yeast counterpart to the $S$. cerevisiae Ddc1/Rad17/Mec3 complex), then Rad17 localization to chromosomes may depend on $\operatorname{Rad} /$ Rad1/Hus1. This notion is consistent with the paradigm of RFC and PCNA. RFC does not interact with DNA in vivo without first forming a binary complex with PCNA (Gomes et al. 2001). By analogy, Rad24 localization may depend on the Ddcl complex in budding yeast.

Absence of significant colocalization between Ddc1 and Rad51 in wild type may reflect a rapid repair of DSBs such that chromosomal overlap of Ddc1 and Rad51 is short lived. The colocalization may also be characteristic of an active checkpoint; Ddc1 may need to be properly modified to remain at the sites of DSB repair. The behavior of Ddc1 raises the possibility that the mammalian checkpoint proteins (e.g., Rad1 and Atr) will show colocalization with Rad51 in mutants defective in meiotic DSB repair.

Substantial but incomplete colocalization between Ddc1 and Rad51 in hop2 mutants suggests that their localization may be sequential and/or transient. Ddc1 and Rad51 may recognize different recombination intermediates, and/or the timing of their action may not be identical. For example, Ddc1 may dissociate from chromosomes as Rad51 is recruited to chromosomes, or vice versa. This notion is supported by the observed differences in the relative intensities of Ddc1 and Rad51 staining at individual foci.

During meiosis $>200$ DSBs are made, but there are only 40-60 Ddc1 foci. However, this does not exclude the possibility that Ddc1 marks all sites of DSB repair. Both Rad51 and Dmc1, known recombination proteins, show only 40-50 foci per nucleus (Bishop 1994). This paradox may reflect (1) asynchronous and/or repair of meiotic DSBs (Borde et al. 2000); (2) one Ddcl focus may represent a cluster of many DSBs; and/or (3) Ddc1 may selectively localize to certain sites of repair (e.g., crossovers vs. non-crossovers, or DSBs with longer ssDNA). 
Ddc1 localization to DSBs requires Rad24, Mec3, and processing of DSBS

The formation of DSBs is required for Ddc1 localization and phosphorylation, suggesting that Ddc1 (and/or its associated proteins) can identify DNA lesions. In rad50S and sae2 strains, in which meiotic DSB processing is impaired, Ddc1 localization is significantly decreased and delayed. One interpretation of this result is that Ddc1 recognizes (albeit inefficiently) unprocessed DSBs (i.e., blunt ends). An alternative possibility is that the phosphorylation and localization of Ddc1 that occurs in rad50S and sae2 strains is attributable to a subset of DSBs escaping normal protection (mediated by covalent attachment of the Spol1 protein) and undergoing degradation to expose long ss tails. This model supposes that Ddc1 binds preferentially to ssDNA or a subsequent recombination intermediate. Consistent with this view, the levels of Ddc1 phosphorylation and localization are much higher in dmc1 strains than in rad50S or sae2 strains, although all three strains accumulate the same number of unrepaired DSBs.

Meiotic DSBs are processed to expose single-stranded tails with $3^{\prime}$ overhangs. It is possible that these intermediates are recognized (directly or indirectly) by Ddc1. An alternative possibility, suggested by the paradigm of RFC and PCNA, is that the Ddc1 complex loads onto recombination intermediates that resemble primed DNA (i.e., intermediates with opposite strand polarity to those generated by the resection of meiotic DSBs). Such intermediates are generated when the ss tails at DSB ends invade homologous sequences in nonsister chromatids. Against this possibility is the observation that such strand invasion intermediates are absent in $d m c 1$ cells (Hunter and Kleckner 2001), yet Ddc1 localizes as intense foci on dmc1 chromosomes.

$\operatorname{Rad} 24$ is also required for proper localization of Ddc1 to meiotic chromosomes. Ddc1 localization is severely impaired, although not completely eliminated, in cells lacking Rad24. This implies that Ddc1 is capable of recognizing DSBs on its own, but its efficiency is greatly improved by Rad24. One possibility is that Rad24 generates ssDNA (Lydall and Weinert 1995), which may aid in Ddc1 localization. However, homology to RFC suggests a more direct role for Rad24 in localizing the Ddc1 complex to meiotic chromosomes; just as RFC is the clamp loader for PCNA, Rad24 may function as a clamp loader for the Ddc1 heterotrimer.

Mec3 is also required for phosphorylation and localization of Ddc1. One possibility is that the integrity of the heterotrimeric complex is required for Ddc1 function, and that the absence of Mec3 disrupts complex formation. The interaction between Ddc1 and Mec3 requires Rad17 (Paciotti et al. 1998), indicating that Ddc1 interacts with Mec3 via Rad17. Paradoxically, however, Rad17 (but not Mec3 or Ddc1) is required for Mek1 phosphorylation (Bailis and Roeder 2000). To explain this discrepancy, we propose that Rad17 normally performs two functions: (1) phosphorylation of Mek1, and (2) modulating the activity of phosphorylated Mek1. The latter ac- tivity requires an intact Ddcl complex, but the former can occur in the absence of Ddc1 or Mec3. Rad17 may have the ability to form a complex on its own (perhaps a homotrimer) that mediates Mek1 phosphorylation. This idea is supported by the recent observation that Rad17 interacts with itself in response to DNA-damage-inducing agents, and this interaction is important for checkpoint function (Zhang et al. 2001). In addition, half of the cellular pool of hRad1 (the human homolog of Rad17) exists in a complex lacking hRad9 and hHus1 (the human homologs of Ddc1 and Mec3, respectively), whereas all of the hRad9 and hHus1 proteins are complexed with hRad1 (Burtelow et al. 2001). These data support the idea that the budding yeast Rad17 protein can function independently of Ddc1 and Mec3.

\section{A role for Rad24 and Ddc1 in recombination}

What roles could Ddc1 and Rad24 play at the sites of DSB repair? First, these proteins may directly process DSBs to propagate the checkpoint signal as proposed previously (Lydall and Weinert 1995). As Rad24 is required for generation of ssDNA (Lydall and Weinert 1995), such processing may amplify the signal and facilitate the localization of additional checkpoint proteins to the sites of DSBs. Second, these proteins may participate in the repair of DSBs. Meiotic recombination takes place preferentially between allelic sequences on nonsister chromatids (for review, see Roeder 1997); however, this bias is weakened in the rad24 mutant. rad24 cells show increased frequencies of sister-chromatid exchange and ectopic recombination between homologous sequences on nonhomologous chromosomes (Grushcow et al. 1999).

A possible role for Ddc1 in DSB repair has also been suggested from studies of the mitotic DNA-damage checkpoint. First, the loss of viability observed in $d d c 1$ cells suffering DNA lesions is not rescued by an artificial delay that allows time for repair (Longhese et al. 1997), indicating that Ddc1 is required for events that contribute to cell viability independent of cell cycle arrest. Second, the $d d c 1$ mutation confers sensitivity to the DNA synthesis inhibitor hydroxyurea (HU), even though Ddc1 is not required for the $S / M$ checkpoint that responds to incomplete DNA replication (Longhese et al. 1997). Sensitivity to HU can be explained if Ddc1 is required for recombinational repair of the stalled replication forks that are generated when DNA synthesis is halted by HU.

\section{Ddc1 functions on a positive feedback loop with respect to Mek1}

How do Rad24 and Ddc1 ensure correct transduction of the checkpoint signal from DSBs? Rad24 is required for Mek1 phosphorylation, indicating that Rad24 has a critical role in activating Mek1 kinase activity (Bailis and Roeder 2000). A previous study showed that Ddcl is not required for Mek1 phosphorylation and suggested that Ddc1 acts downstream of Red1 (Bailis and Roeder 2000). Here, we revisit this placement of Ddcl with respect to 
Mek1 and Red1. Ddc1 is required for Mek1-dependent phosphorylation of Red1, indicating that Ddc1 functions upstream of Red1 phosphorylation. Consistent with this finding, the status of Red1 phosphorylation has no effect on Ddc1 localization, although the presence of Red1 on chromosomes is important for this process.

Several observations suggest that Ddc1 acts together with Mek1. First, Ddc1 colocalizes with Mek1 on meiotic chromosomes in strains where the checkpoint is activated. Second, the proper localization of Mek1 to meiotic chromosomes requires Ddc1. Third, Mek1-mediated phosphorylation of Red1 requires Ddc1. Finally, Ddc1 phosphorylation depends on Mek1 (although its chromosomal localization is independent of Mek1), raising the possibility that Mek1 directly phosphorylates Ddc1. Given that the phosphorylation of Mek1 depends on Mec1 (Bailis and Roeder 2000), the requirement for Mec1 in Ddc1 phosphorylation (Paciotti et al. 1998) may simply reflect the role of Mec1 in activation of Mek1. This linear relationship diverges from that described in the DNA-damage checkpoint, in which Ddc1 phosphorylation depends on Mec1, and Ddc1 is required for phosphorylation of Rad53 (a mitotic paralog of Mek1; Paciotti et al. 1998).

The mutual dependence of Ddc1 phosphorylation and Mek1 activity suggests that Ddc1 operates in a positive feedback loop that promotes Mek1 function. How can Ddc1 promote Mek1-mediated phosphorylation of Red1? One possibility is that Ddc1 is required for Mek1 to associate stably with chromosomes, where Redl and perhaps other relevant substrates reside. The localization of Mek1 to chromosomes is delayed and inefficient in the absence of Ddc1. Furthermore, although many Mek1 foci accumulate on chromosomes by pachytene, the intensity of these foci is lower than that seen in the control strain. This decrease in the intensity of Mek1 foci suggests that the Ddc1 complex anchors Mek1 to meiotic chromosomes until recombination intermediates are repaired, analogous to the anchoring of DNA polymerases by PCNA (for review, see Tsurimoto 1998).

It is also possible that the reduced localization of Mek1 to chromosomes in $d d c 1$ cells is more than adequate to carry out Mek1 function. This idea is consistent with the finding that Mek1 becomes phosphorylated in the $d d c 1$ mutant, and this phosphorylation is presumed to occur on meiotic chromosomes (Bailis and Roeder 2000). However, phosphorylation of Mek1 may be delayed in $d d c 1$ cells (as a consequence of delayed localization), which may perturb the timing of kinasesubstrate interactions that are essential for proper transduction of the checkpoint signal. In addition, although $d d c 1$ has no effect on the phosphorylation pattern of Mek1 as detected in gel shift assays (Bailis and Roeder 2000), it is possible that Ddc1 is required for phosphorylation of a critical residue in Mek1, which could affect its kinase activity. Alternatively, Ddc1 could influence Mek1 by promoting a conformational change and/or facilitating correct substrate recognition. In this case, impaired Mek1 localization to chromosomes might be an indirect consequence of the failure of Mek1 to undergo conformational changes mediated by Ddc1. Interestingly, PCNA is capable of stimulating the in vitro activity of the yeast analog of the FEN-1 (yFEN-1) endonuclease (Li et al. 1995). Through protein-protein interaction, PCNA focuses yFEN-1 on its substrates, thereby stimulating its activity by 10 - to 50 -fold. This paradigm of PCNA and yFEN-1 raises the possibility that the Ddc1 complex stimulates Mek1 kinase activity via a direct physical interaction.

What could be the role of Ddc1 phosphorylation? A correlation between the extent of phosphorylation and the degree of meiotic arrest implies that this modification is important for the checkpoint function. Phosphorylation is not required for Ddc1 to localize or remain on meiotic chromosomes, because Ddc1 localization is unaffected in mek 1 mutants. This also implies that phosphorylation is not necessary for maintaining the integrity of the heterotrimeric complex, given that the formation of the complex seems to be necessary for Ddc1 localization to meiotic chromosomes. We speculate that phosphorylation of Ddc1 is required for mediating the proper activity of Mek1, either by a direct enzymatic activation of Mek1 (similar to PCNA and yFEN-1) or by anchoring Mek1 to chromosomes.

How does Redl affect the pachytene checkpoint? The requirement for Red1 for Ddc1 localization suggests that Red1 directly recruits Ddc1 to meiotic chromosomes. However, because Red1 is required for the integrity of the axial cores of meiotic chromosomes (for review, see Roeder 1997) and for the localization of numerous checkpoint proteins (e.g., Ddc1 and Mek1; this study; Bailis and Roeder 1998), we favor the view that Redl carries out a more general role in creating a favorable environment for checkpoint proteins (Xu et al. 1997). Red1 may provide the chromatin structure necessary for checkpoint proteins to identify DNA lesions and properly interact with each other to transduce the checkpoint signal. Another possibility is that Red1 phosphorylation serves as an indicator of ongoing DSB repair that is recognized by the checkpoint (Bailis and Roeder 2000). However, the presence of checkpoint proteins that function upstream of Red1 phosphorylation that also respond directly to the presence of DNA lesions makes it difficult to imagine a need for a secondary sensor of recombination status. Whether transduction of the checkpoint signal occurs via phosphorylation of Red1 or Mek1-dependent phosphorylation of other proteins remains to be determined.

\section{Summary}

In conclusion, we propose that Rad24 and Ddc1 function as components of the checkpoint machinery that recognize meiotic DSBs and as modulators of Mek1 kinase activity, thereby ensuring correct transduction of the checkpoint signal from Mek1 to its substrate (Fig. 7B). DSBs may be recognized by Rad24, which loads the Ddc1 complex onto sites of DSB repair. At the same time, Rad24 facilitates phosphorylation of Mek1. Ddc1, which is at the same chromosomal locations as Mek1, acts to 
anchor Mek1 to meiotic chromosomes, and promotes phosphorylation of Red1 and possibly other substrates. This places Ddc1 on a positive feedback loop with respect to Mek1, in which Ddc1 serves both as a substrate and a mediator of Mek1 kinase activity.

\section{Materials and methods}

\section{Genetic methods}

Yeast manipulations were carried out as described (Bailis and Roeder 2000), except that sporulation was carried out at $31^{\circ} \mathrm{C}$. Spore viability was determined by tetrad dissection; the number of spores scored was 96 for wild-type cells, 96 for zip 1 cells, 512 for $d d c 1$ cells, 452 for zip1 $d d c 1$ cells, and 104 for DDC1-myc cells.

A screen to find mutations that allow the zip1 mutant to sporulate was carried out as described by San-Segundo and Roeder (1999) with the following modifications. Transformants carrying transposon insertions were scored for sporulation both by using a fluorescence assay and by monitoring the expression of a ysw1::1acZ fusion gene (Tung et al. 2000). Linkage between the mutant phenotype and the transposon was established by transforming the starting strain with rescued plasmid containing the transposon and flanking sequences on both sides. Plasmid rescue (Ross-Macdonald et al. 1997) was carried out using plasmid EB89, which was made by subcloning a BglII-SacI fragment containing the KanMX4 module (Wach et al. 1994) into pRSQ (Ross-Macdonald et al. 1997). pEB89 was targeted for integration by cutting with Bsu36I, and the plasmid and transposon were then released by cutting with BgIII.

\section{Strains and plasmids}

All strains are isogenic with S3246, which is a MATa /MAT $\alpha$ diploid homozygous for his4-260 leu2-3, 112 thr1-4 trp1-289 ura3-1 ade2-1. S3246 was constructed by mating BR1919-8B (Rockmill and Roeder 1990) to a MATa derivative of BR1919-8B (Rockmill et al. 1995). Diploids used in this study were made by mating appropriate haploids, generated by transformation and/ or genetic crosses.

Ddc1 was tagged at its genomic locus (after the 317 th codon) with three copies of the myc epitope using a PCR strategy (Schneider et al. 1995). Mek1 was also tagged at its genomic locus (before the Stop codon) with super glow GFP by a PCR strategy (Schneider et al. 1995; Agarwal and Roeder 2000). Super glow GFP was amplified using a plasmid provided by J-.Y. Leu (Harvard University, Boston, MA). The hop2::ADE2 mutant was made by transforming with plasmid pEB30, which was constructed as follows. First, a hop2 deletion was constructed by removing 416 bp between the AvaI and AccI sites of pEB3, which contains the full-length $H O P 2$ gene. The resulting deletion mutant was inserted between the Pst I and Not I sites of pBluescript SK(+) to generate pEB4. A BglII fragment containing $A D E 2$ was then inserted at the BamHI site in the hop2 gene in pEB4 to generate pEB30. pEB30 was targeted for substitutive transformation by digesting with NheI and SpeI.

The glc7-T152K mutation was introduced as follows. First, the BamHI-HindIII fragment containing GLC7 from pJTL18T152K (Bailis and Roeder 2000) was inserted between the BamHI and HindIII sites of the URA3-containing plasmid YIplac211 (Geitz and Sugino 1988). The mutation was introduced into yeast by two-step transplacement (Rothstein 1991).
The plasmid was cut with BgIII prior to yeast transformation to target integration at the GLC7 locus.

Plasmids for introducing the following mutations have been described: ndt80::LEU2 and ndt80::URA3 (Tung et al. 2000), zip1::LEU2 (Sym et al. 1993), spo11::ADE2 (Smith and Roeder 1997), mek1::1acZ-LEU2 (Bailis and Roeder 1998), red1::URA3 (Smith and Roeder 1997), rad24::TRP1 (Lydall and Weinert 1997), mec3::TRP1 (Lydall and Weinert 1997), and rad50K181::URA3 (Alani et al. 1990). rad50-K181::URA3 is referred to as rad50S elsewhere in the text. BR1919-8B strains carrying $d d c 1:: A D E 2$ and $d m c 1:: U R A 3$ disruptions were obtained from B. Rockmill and J. Novak (Yale University, New Haven, CT), respectively.

\section{Two-hybrid protein assays}

Construction of plasmids containing MSH5 and RAD57 fused to the GAL4 DNA-binding domain have been described (Agarwal and Roeder 2000). SAE1, SAE3, and DDC1 were fused to the Gal4 DNA-binding domain as follows: the SAE1 and SAE3 ORFs were amplified by PCR using pNKY1197 and pNKY1193, respectively, as templates (McKee and Kleckner 1997). The DDC1 ORF was amplified by PCR using yeast genomic DNA as a template. Using appropriate primers, a BamHI site was introduced just before the Start codon of each ORF, and a SalI site was inserted just before each Stop codon. The resulting BamHISall fragments were inserted at the BamHI-SalI site of pBG4D-1 (Bailis and Roeder 1998) to generate pR1880 (Sae1), pR1884 (Sae3), and pR1848 (Ddc1; provided by B. Rockmill). RAD24 fused to the GAL4 transcriptional activation domain was made as follows: the RAD24 ORF was amplified by PCR to introduce an XmaI site just before the Start codon and a SalI site just before the Stop codon. The resulting fragment was inserted at the XmaI-XhoI site of pACTII (Bailis and Roeder 1998) to generate $\mathrm{pEB} 38$.

Two hybrid assays were carried out in a diploid yeast strain derived from Y187 and Y190 (Harper et al. 1993). The interaction was assayed via a $\beta$-galactosidase reporter under the control of a $G A L$ promoter. $\beta$-Galactosidase units are defined as amount of $o$-nitrophenyl- $\beta$-D-galactopyranoside cleaved per minute per liter of cells (Bartel and Fields 1995). Meiotic two-hybrid assays were carried out after sporulation at $30^{\circ} \mathrm{C}$ for $9 \mathrm{~h}$.

\section{Immunoprecipitations and Western blot analysis}

Preparation of yeast cell extracts, Western blotting, and CIP treatment were performed as described (Tung et al. 2000) with the following modifications. Each immunoprecipitation was carried out with extract derived from $250 \mathrm{~mL}$ of sporulating cells, using mouse anti-myc antibody (Covance) or rabbit antiRed1 antibody (Smith and Roeder 1997) at 1:100 dilution. Immunoprecipitated Ddc1 was fractionated by $8 \%$ SDS-PAGE for $24 \mathrm{~h}$ at $15 \mathrm{~V}$. Red1 protein was fractionated by $6 \%$ SDS-PAGE for $20 \mathrm{~h}$ at $15 \mathrm{~V}$.

\section{Cytology}

To determine the frequency and kinetics of meiotic nuclear division, cells were fixed in $70 \%$ ethanol and frozen at $-20^{\circ} \mathrm{C}$ prior to staining with DAPI. Cells were observed and counted under the fluorescent microscope. The values plotted in Figure 1 represent at least 200 nuclei per strain at each time point.

Spread meiotic nuclei were prepared and stained with antibodies as described (Sym et al. 1993) except that fetal bovine serum (GIBCO BRL) was used for blocking slides. Antibodies were preabsorbed with spheroplasted yeast cells undergoing 
sporulation. Mouse anti-myc antibody (Covance), rabbit antiZip1 antibody (Sym et al. 1993), rabbit anti-Red1 antibody (Smith and Roeder 1997), rabbit anti-GFP antibody $(1 \mathrm{mg} / \mathrm{mL}$; Agarwal and Roeder 2000), rat anti-tubulin antibody (Sera-Lab), and rabbit anti-Rad51 antibody (a gift of Akira Shinohara, University of Chicago, IL) were used at 1:100 dilution. Donkey antirat antibody conjugated to $\mathrm{Cy} 3$, donkey anti-rabbit antibody conjugated to FITC or $\mathrm{Cy} 3$, and donkey anti-mouse antibody conjugated to Cy3 (all from Jackson Immuno Research Labs) were used at 1:200 dilution.

Nuclei at different stages of meiotic prophase were identified based on staining spread chromosomes with antibodies to Zip1, Red1, or tubulin. For strains in which synapsis occurs normally, nuclei were categorized as zygotene if Zip1 staining was dotty and as pachytene if Zip1 staining was linear. For strains defective in synapsis, nuclei were categorized as zygotene if Red1 staining was dotty and as pachytene if Redl staining was linear. For the zip1 red1 double mutant and the corresponding control strain (Fig. 6E), nuclei were stained with anti-tubulin antibodies, and nuclei in meiotic prophase were identified based on the presence of duplicated but unseparated spindle pole bodies.

A Nikon E800 microscope and an Olympus IX70 DeltaVision microscope (each equipped with a 100× objective lens and fluorescent optics) were used to observe antibody-stained meiotic chromosomes. Images were captured by Photometrics Imagepoint and Quantix CCD cameras.

To quantitate Ddc1 foci on meiotic chromosomes, the following numbers of nuclei were examined: 65 wild type, 83 zip1, 20 ndt80, 20 dmc1, 13 rad50S, 15 spo11, 56 zip1 rad24, 40 zip1 mec3, 26 zip1 red1, and 98 zip1 mek1. To quantitate Mek1 foci on meiotic chromosomes, the following numbers of nuclei were examined: $11 d m c 1$ ndt80 (heterozygous for $d d c 1$ ), $14 d m c 1$ $n d t 80 d d c 1$, and $9 d m c 1$ ndt 80 mec3. Standard errors were calculated as described (Zar 1999). Student $t$-tests were performed to compare the number of Ddc1 foci in wild-type zygotene versus wild-type pachytene, wild-type zygotene versus zip1 pachytene, wild-type pachytene versus zip1 pachytene, spo11 versus rad50S, zip1 rad24 versus zip1 mec3, and zip1 versus zip1 mek1. For the latter three pairwise comparisons, there were no significant differences between zygotene and pachytene nuclei. Data were therefore pooled to generate the histograms shown in Figures 4C and 6D. The number of Ddcl foci in the pairs of samples listed are different from each other with $\alpha$ set at 0.05 (Zar 1999).

To determine the amount of overlap between Ddc1 and Rad51, and between Ddc1 and Mek1, the total number of Ddc1 foci per spread nucleus was determined and compared with the number of Ddc1 foci containing either Rad51 or Mek1 foci. The same assay was used to calculate the percent of Rad51 or Mek1 foci containing Ddc1 foci. Fortuitous colocalization was determined by rotating an overlay corresponding to either the Rad51 or the Mek 1 signal by $90^{\circ}$ or $180^{\circ}$ and quantitating the overlap between the misoriented images as described (Gasior et al. 1998). At least 10 spread nuclei from each strain were used for quantitation.

\section{Acknowledgments}

We thank D. Bishop, N. Kleckner, J. Novak, A. Shinohara, P. Sung, B. Rockmill, and T. Weinert for providing plasmids, yeast strains, and antibodies. We thank J. Bailis, J. Fung, and B. Rockmill for comments on the manuscript. E.-J.E.H. especially thanks J-.Y. Leu and L. Maloisel for helpful discussions, and R. Shew and N. Shapiro for technical assistance. This work was supported by the Howard Hughes Medical Institute and by National Institutes of Health grant GM28904 to G.S.R.
The publication costs of this article were defrayed in part by payment of page charges. This article must therefore be hereby marked "advertisement" in accordance with 18 USC section 1734 solely to indicate this fact.

\section{References}

Agarwal, S. and Roeder, G.S. 2000. Zip3 provides a link between recombination enzymes and synaptonemal complex proteins. Cell 102: 245-255.

Alani, E., Padmore, R., and Kleckner, N. 1990. Analysis of wildtype and rad50 mutants of yeast suggests an intimate relationship between meiotic chromosome synapsis and recombination. Cell 61: 419-436.

Bailis, J.M. and Roeder, G.S. 1998. Synaptonemal complex morphogenesis and sister-chromatid cohesion require Mek1-dependent phosphorylation of a meiotic chromosomal protein. Genes \& Dev. 12: 3551-3563.

- 2000. Pachytene exit controlled by reversal of Mek1dependent phosphorylation. Cell 101: 211-221.

Bartel, P. and Fields, S. 1995. Analyzing protein-protein interactions using two-hybrid system. Meth. Enzymol. 254: 241263.

Bishop, D.K. 1994. RecA homologs Dmc1 and Rad51 interact to form multiple nuclear complexes prior to meiotic chromosome synapsis. Cell 79: 1081-1092.

Bishop, D.K., Park, D., Xu, L., and Kleckner, N. 1992. DMC1: A meiosis-specific yeast homolog of E. coli recA required for recombination, synaptonemal complex formation, and cell cycle progression. Cell 69: 439-456.

Borde, V., Goldman, A.S.H., and Lichten, M. 2000. Direct coupling between meiotic DNA replication and recombination initiation. Science 290: 806-809.

Burtelow, M.A., Roos-Mattjus, P.M.K., Rauen, M., Babendure, J.R., and Karnitz, L.M. 2001. Reconstitution and molecular analysis of the hRad9-hHus1-hRad1 (9-1-1) DNA damage responsive checkpoint complex. J. Biol. Chem. 276: 2590325909.

Chu, S. and Herskowitz, I. 1998. Gametogenesis in yeast is regulated by a transcriptional cascade dependent on $\mathrm{Ndt} 80$. Mol. Cell 1: 685-696.

Elledge, S.J. 1996. Cell cycle checkpoints: Preventing an identity crisis. Science 274: 1664-1672.

Gasior, S.L., Wong, A.K., Kora, Y., Shinohara, A., and Bishop, D.K. 1998. Rad52 associates with RPA and functions with Rad55 and Rad57 to assemble meiotic recombination complexes. Genes \& Dev. 12: 2208-2221.

Geitz, R.D. and Sugino, A. 1988. New yeast-Escherichia coli shuttle vectors constructed with in vitro mutagenized yeast genes lacking six-base pair restriction sites. Gene 74: 527534.

Gomes, X., Schmidt, S.L.G., and Burgers, P.M.J. 2001. ATP utilization by yeast replication factor C. II. Multiple stepwise ATP binding events are required to load proliferating cell nuclear antigen onto primed DNA. I. Biol. Chem. 276: 34776-34783.

Grushcow, J.M., Holzen, T.M., Park, K.J., Weinert, T., Lichten, M., and Bishop, D.K. 1999. Saccharomyces cerevisiae checkpoint genes $M E C 1, R A D 17$ and $R A D 24$ are required for normal meiotic recombination partner choice. Genetics 153: 607-620.

Harper, J.W., Adami, G.R., Wei, N., Keyomarsi, K., and Elledge, S.J. 1993. The p21 cdk-interacting protein Cip1 is a potent inhibitor of G1 cyclin-dependent kinases. Cell 75: 805-816. 
Hunter, N. and Kleckner, N. 2001. The single-end invasion: An asymmetric intermediate at the double-strand break to double-Holliday junction transition of meiotic recombination. Cell 106: 59-70.

Kai, M., Tanaka, H., and Wang, T.S.-F. 2001. Fission yeast Rad17 associates with chromatin in response to aberrant genomic structures. Mol. Cell. Biol. 21: 3289-3301.

Kondo, T., Wakayama, T., Naiki, T., Matsumoto, K., and Sugimoto, K. 2001. Recruitment of Mec1 and Ddcl checkpoint proteins to double-strand breaks through distinct mechanisms. Science 294: 867-870.

Leu, J.-Y., Chua, P.R., and Roeder, G.S. 1998. The meiosis-specific Hop2 protein of $S$. cerevisiae ensures synapsis between homologous chromosomes. Cell 94: 375-386.

Li, X., Li, J., Harrington, J., Lieder, M.R., and Burgers, P.M.J. 1995. Lagging strand DNA synthesis at the eukaryotic replication fork involves binding and stimulation of FEN-1 by proliferating cell nuclear antigen. J. Biol. Chem. 270: 2210922112.

Longhese, M.P., Paciotti, V., Fraschini, R., Zaccarini, R., Plevani, P., and Lucchini, G. 1997. The novel DNA damage checkpoint protein Ddclp is phosphorylated periodically during the cell cycle and in response to DNA damage in budding yeast. EMBO J. 16: 5216-5226.

Lydall, D. and Weinert, T. 1995. Yeast checkpoint genes in DNA damage processing: Implications for repair and arrest. Science 270: 1488-1491.

-1997. G2/M checkpoint genes of Saccharomyces cerevisiae: Further evidence for roles in DNA replication and/or repair. Mol. Gen. Genet. 256: 638-651.

Lydall, D., Nikolsky, Y., Bishop, D.K., and Weinert, T. 1996. A meiotic recombination checkpoint controlled by mitotic checkpoint genes. Nature 383: 840-843.

McKee, A.H.Z. and Kleckner, N. 1997. Mutations in Saccharomyces cerevisiae that block meiotic prophase chromosome metabolism and confer cell cycle arrest at pachytene identify two new meiosis-specific genes $S A E 1$ and $S A E 3$. Genetics 146: $817-834$.

Naiki, T., Shimomura, T., Kondo, T., Matsumoto, K., and Sugimoto, K. 2000. Rfc5, in cooperation with $\operatorname{Rad} 24$, controls DNA damage checkpoints throughout the cell cycle in Saccharomyces cerevisiae. Mol. Cell. Biol. 20: 5888-5896.

Paciotti, V., Lucchini, G., Plevani, P., and Longhese, M.P. 1998. Meclp is essential for phosphorylation of the yeast DNA damage checkpoint protein Ddclp, which physically interacts with Mec3p. EMBO J. 17: 4199-4209.

Rauen, M., Burtelow, M.A., Dufault, V.M., and Karnitz, L.M. 2000. The human checkpoint protein hRad17 interacts with the PCNA-like proteins hRad1, hHus1, and hRad9. J. Biol. Chem. 275: 29767-29771.

Rockmill, B. and Roeder, G.S. 1990. Meiosis in asynaptic yeast. Genetics 126: 563-574.

Rockmill, B., Sym, M., Scherthan, H., and Roeder, G.S. 1995. Roles for two RecA homologs in promoting meiotic chromosome synapsis. Genes \& Dev. 9: 2684-2695.

Roeder, G.S. 1997. Meiotic chromosomes: It takes two to tango. Genes \& Dev. 11: 2600-2621.

Roeder, G.S. and Bailis, J.M. 2000. The pachytene checkpoint. Trends Genet. 16: 395-403.

Ross-Macdonald, P., Sheehan, A., Roeder, G.S., and Snyder, M. 1997. A multipurpose transposon system for analyzing protein production, localization, and function in Saccharomyces cerevisiae. Proc. Natl. Acad. Sci. 94: 190-195.

Rothstein, R. 1991. Targeting, disruption, replacement and allele rescue: Integrative DNA transformation in yeast. Meth. Enzymol. 194: 281-301.
San-Segundo, P. and Roeder, G.S. 1999. Pch2 links chromatin silencing to meiotic checkpoint control. Cell 97: 313-324.

Schneider, B.L., Seufert, W., Steiner, B., Yang, Q.H., and Futcher, A.B. 1995. Use of polymerase chain reaction epitope tagging for protein tagging in Saccharomyces cerevisiae. Yeast 11: 1265-1274.

Smith, A.V. and Roeder, G.S. 1997. The yeast Red1 protein localizes to the cores of meiotic chromosomes. J. Cell Biol. 136: 957-967.

Storlazzi, A., Xu, L., Schwacha, A., and Kleckner, N. 1996. Synaptonemal complex (SC) component Zip1 plays a role in meiotic recombination independent of SC polymerization along the chromosomes. Proc. Natl. Acad. Sci. 93: 90439048.

Sym, M., Engebrecht, J., and Roeder, G.S. 1993. ZIP1 is a synaptonemal complex protein required for meiotic chromosome synapsis. Cell 72: 365-378.

Tsurimoto, T. 1998. PCNA, a multifunctional ring on DNA. Biochim. Biophys. Acta 1443: 23-39.

Tung, K.-S., Hong, E., and Roeder, G.S. 2000. The pachytene checkpoint prevents accumulation and phosphorylation of the meiosis-specific transcription factor Ndt80. Proc. Natl. Acad. Sci. 97: 12187-12192.

Venclovas, C. and Thelen, M. 2000. Structure-based predictions of Rad1, Rad9, Hus1 and Rad17 participation in sliding clamp and clamp-loading complexes. Nucleic Acids Res. 28: 2481-2493.

Wach, A., Brachat, A., Pohlmann, R., and Philippsen, P. 1994. New heterologous modules for classical or PCR-based gene disruptions in Saccharomyces cerevisiae. Yeast 10: 17931808.

Weinert, T. 1998. DNA damage checkpoints update: Getting molecular. Curr. Opin. Genet. Dev. 8: 185-193.

$\mathrm{Xu}$, L., Weiner, B.M., and Kleckner, N. 1997. Meiotic cells monitor the status of the interhomolog recombination complex. Genes \& Dev. 11: 106-118.

Zar, J. 1999. Biostatistical analysis, 4th ed. Prentice Hall, Upper Saddle River, NJ.

Zhang, H., Zhu, Z., Vidanes, G., Mbangkollo, D., Liu, Y., and Siede, W. 2001. Characterization of DNA damage-stimulated self-interaction of Saccharomyces cerevisiae checkpoint protein Rad17p. J. Biol. Chem. 276: 26715-26723.

Zhou, B.-B.S. and Elledge, S.J. 2000. The DNA damage response: Putting checkpoints in perspective. Nature 408: 433-439. 


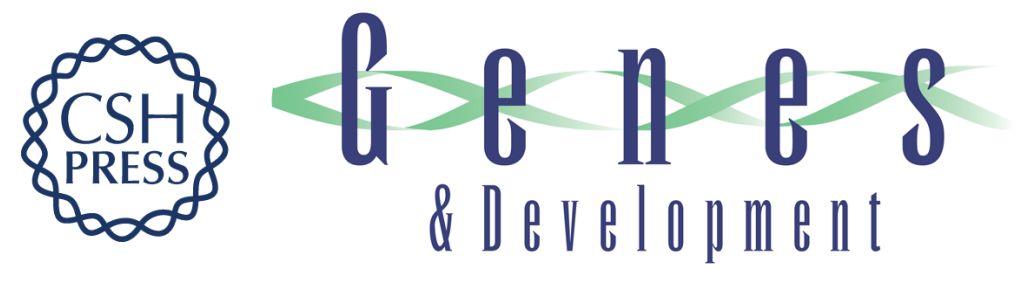

\section{A role for Ddc1 in signaling meiotic double-strand breaks at the pachytene checkpoint}

Eun-Jin Erica Hong and G. Shirleen Roeder

Genes Dev. 2002, 16:

Access the most recent version at doi:10.1101/gad.938102

References This article cites 48 articles, 25 of which can be accessed free at: http://genesdev.cshlp.org/content/16/3/363.full.html\#ref-list-1

License

Email Alerting

Receive free email alerts when new articles cite this article - sign up in the box at the top Service right corner of the article or click here.

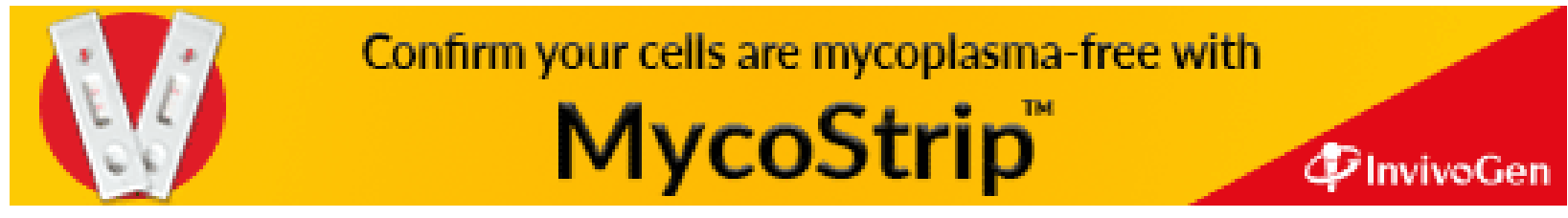

